\title{
Uso de Operações SIMD em uma Biblioteca de Algoritmos Bio-inspirados*
}

\author{
Natiele Lucca $^{1}$, Claudio Schepke ${ }^{1}$ \\ ${ }^{1}$ Ciência da Computação - Universidade Federal do Pampa (UNIPAMPA) \\ Alegrete - RS - Brazil \\ natielelucca@gmail.com, claudioschepke@unipampa.edu.br
}

\begin{abstract}
Resumo. Uma estratégia para modelar algoritmicamente um problema é utilizar conceitos de computação natural, também conhecida como computação bioinspirada. Neste trabalho é proposta uma biblioteca open source bio-inspirada que utiliza instruções SIMD. Com os resultados dos testes, comprovou-se que a versão paralela dos algoritmos desenvolvidos mantém a qualidade da solução e reduz o tempo de execução.
\end{abstract}

\section{Introdução}

A resolução de um problema pode não ser alcançada de forma exata devido a complexidade dada por um número elevado de variáveis e/ou soluções potenciais. Um algoritmo que descreve um problema sequencialmente pode demandar de uma grande capacidade de processamento para encontrar a solução ótima [Ignácio and Ferreira 2002]. O tempo exigido para a computação exata e sequencial varia de horas à anos, o que destaca a importância de abordagens e técnicas que otimizem a execução de algoritmos [Nievergelt et al. 1995]. Os problemas citados anteriormente podem ser identificados em diversas áreas de pesquisa, como em simulações de atividades reais que envolvem engenharia, ciência e economia.

Nesse contexto, algoritmos bioinspirados são uma estratégia para reduzir o tempo de execução, de maneira que a solução seja mantida ou melhorada. Estes aplicam técnicas de inteligência de enxames ou inteligência de colônias, ou ainda inteligência coletiva, que simulam o comportamento coletivo de sistemas auto-organizados, distribuídos, autônomos, flexíveis e dinâmicos [Serapiao 2009]. Os elementos que integram o enxame ou colônia são capazes de otimizar um objetivo global através da busca colaborativa em um espaço seguindo regras específicas [Kennedy and Eberhart 2001].

O objetivo deste trabalho é aplicar a concorrência em unidades vetoriais com operações SIMD (Single Instruction Multiple Data) na biblioteca bio-inspirada ${ }^{1}$ desenvolvida pelos autores, a fim de diminuir o tempo de execução e auxiliar os desenvolvedores na solução de problemas. A biblioteca implementa os algoritmos clássicos: Enxame de Partículas (PSO - Particle Swarm Optimization); Colônia Artificial de Abelhas (ABC - Artificial Bee Colony); e Colônias de Formigas (ACO - Ant Colony Optimization).

O artigo está organizado da seguinte forma. A Seção 2 traz os métodos e filtros utilizados para a realização deste trabalho. Na Seção 3 são apresentados os resultados. Por fim, na Seção 4, são destacadas as considerações finais sobre a pesquisa realizada.

\footnotetext{
* O presente trabalho foi desenvolvido no Laboratório de Estudos Avançados (LEA) com apoio do PDA Iniciação Científica/Unipampa 2018 e PROBIC/FAPERGS 2018 e 2019.

${ }^{1}$ Disponível em https://github.com/NatiLucca/Bio-inspired-library
} 


\section{Metodologia}

Para validar a implementação é preciso verificar as características dos algoritmos através de testes. Os testes são realizados por funções que possuem propriedades diversas, para garantir que o algoritmo possa ou não resolver certos tipos de otimização com eficiência [Yang 2010]. Para testar os algoritmos implementados na biblioteca bio-inspirada foi desenvolvido um benchmark, com uma aplicação base composta por sete funções de teste clássicas ${ }^{2}$ : Alpine, Booth, Easom, Griewank, Rastrigin, Rosenbrock e Sphere.

Os algoritmos foram desenvolvidos a partir do respectivo pseudocódigo disponível na literatura. Em seguida, diretivas da API OpenMP foram investigadas a fim de verificar qual ou quais são mais adequadas para cada algoritmo. Para comparar as versões sequenciais e paralelas foram gerados arquivos que contém os valores das entradas. Dessa forma, ambas as versões foram submetidas ao mesmo caso de teste. Uma execução compreende a geração dos arquivos de entrada, a execução da função sequencialmente e a execução da função paralela.

A versão sequencial e paralela leem dos arquivos de entrada a população inicial e os demais valores aleatórios do código. A versão paralela contém as mesmas instruções da versão sequencial, tendo apenas o acréscimo das diretivas OpenMP. Ambas as versões retornam o melhor valor encontrado pelo algoritmo e o tempo de execução em segundos. A biblioteca também dispõem de uma versão que retorna além da solução e do tempo, todos os valores utilizados na execução dos testes.

Para medir o desempenho das versões dos algoritmos paralelos, foi utilizado o conceito de speedup $(S)$. O speedup é definido como a razão entre o tempo de computação do algoritmo serial ( $\left.T_{\text {serial }}\right)$ e o tempo de computação do algoritmo paralelo $\left(T_{\text {paralelo }}\right)$. Uma outra forma de mensurar o quanto uma versão paralela é melhor que a versão sequencial é considerar o percentual de ganho de desempenho apresentado na Equação 1.

$$
S=\frac{T_{\text {serial }}-T_{\text {paralelo }}}{T_{\text {paralelo }}} * 100=\left(\frac{T_{\text {serial }}}{T_{\text {paralelo }}}-1\right) * 100
$$

Todos os testes foram realizados na workstation da Universidade Federal do Pampa (UNIPAMPA), campus Alegrete que possui a seguinte configuração: dois processadores Intel ${ }^{\circledR}$ Core $^{\mathrm{TM}}$ Xeon CPU E5-2650, com 2,0 GHz de frequência, 8 núcleos e 16 threads, 128 GB de memória RAM e com sistema operacional Ubuntu na versão 16.04 de 64 bits. Os resultados são calculados a partir da média de 30 execuções.

\section{Resultados}

Após a implementação sequencial da biblioteca e verificação do código, foram feitos testes para avaliar o tempo de execução das implementações. Desta forma, foi possível obter um baseline, o qual foi tomado como referência para efeito de comparação dos tempos de execução das implementações paralelas.

A diretiva \#pragma omp simd foi utilizada, pois, essa diretiva permite usar unidades vetoriais, onde um bloco de instruções é executado simultaneamente. Como uma instrução é executada sobre diferentes dados, para garantir a eficiência desse método

\footnotetext{
${ }^{2}$ As funções e as suas respectivas características estão disponíveis em http://benchmarkfcns.xyz/fcns
} 
Tabela 1. Casos de Teste - Melhores Resultados.

\begin{tabular}{|c|c|c|c|c|c|c|}
\hline Função & Solução & $\begin{array}{c}\text { ABC } \\
\text { T. Desemp. }\end{array}$ & Des. Padrão & Solução & $\begin{array}{c}\text { PSO } \\
\text { T. Desemp. }\end{array}$ & Des. Padrão \\
\hline 1. Alpine & $0,00 \mathrm{E}+00$ & 35,59 & 0,0062 & $1,95 \mathrm{E}+00$ & 301,01 & 0,0195 \\
\hline 2. Booth & $1,30 \mathrm{E}-17$ & 30,38 & 0,0017 & $1,47 \mathrm{E}-05$ & 602,84 & 0,0002 \\
\hline 3. Easom & $-1,00 \mathrm{E}+00$ & 41,32 & 0,0094 & $-9,64 \mathrm{E}-01$ & 289,20 & 0,0013 \\
\hline 4. Griewank & $1,11 \mathrm{E}-13$ & 53,63 & 0,0088 & $1,20 \mathrm{E}+02$ & 224,88 & 0,1064 \\
\hline 5. Rastrigin & $5,35 \mathrm{E}-13$ & 63,34 & 0,0131 & $3,05 \mathrm{E}+02$ & 276,75 & 0,1123 \\
\hline 6. Rosenbrock & $9,16 \mathrm{E}+00$ & 140,83 & 0,0118 & $1,30 E+05$ & 2007,38 & 0,0711 \\
\hline 7. Sphere & $1,04 \mathrm{E}-17$ & 35,65 & 0,0054 & $1,31 \mathrm{E}-02$ & 1141,37 & 0,0027 \\
\hline
\end{tabular}

de paralelismo os endereços acessados no laço de repetição devem estar consecutivos em memória. Desse modo, a alocação de memória contígua é aplicada na implementação dos algoritmos sobre os vetores dos dados. Dos resultados obtidos pela diretiva \#pragma omp simd, nenhum resultado foi negativo, ou seja, o tempo de execução paralelo foi sempre inferior ao tempo de execução sequencial em todos os casos.

Os testes realizados ${ }^{3}$ retornaram a mesma solução ótima para as versões sequencial e paralela, pois ambas eram submetidas ao mesmo caso de teste. A Tabela 1 apresenta para cada algoritmo as soluções médias obtidas a taxa de desempenho em (\%) e o desvio padrão para cada respectiva função de teste.

A Tabela 1 apresenta a média dos valores de cada uma das 30 execuções para cada função e algoritmo. Como são realizados testes sobre execuções sequenciais e paralelas os resultados são comparados, pois como os ambas as versões são submetidas aos mesmos valores o resultados deve ser igual. Para todos os testes realizados os resultados obtidos foram iguais. Os valores ótimos para as funções são $-1,00 E+00$ para a função Easom e $0,00 E+00$ para as demais funções.

$\mathrm{O}$ algoritmo $\mathrm{ABC}$ obteve as soluções ótimas para as funções 1 e 3, Alpine e Easom, respectivamente. As funções Booth e Sphere obtiveram bons resultados, sendo que a primeira é bidimensional e a segunda possui 6 dimensões. As funções Griewank e Rastrigin também obtiveram boas soluções. Essas funções possuem 50 dimensões. A pior solução encontrada pelo algoritmo foi a Rosenbrock que também possui 50 dimensões. O algoritmo $\mathrm{ABC}$ obteve soluções ótimas e soluções médias próximas à ótima. Dessa forma, analisando as soluções, é um algoritmo que obtém bons resultados aplicado a diferentes funções, sendo que essas funções possuem características diferentes.

O algoritmo PSO obteve soluções próximas as ótimas. As funções Alpine, Booth, Easom e Sphere possuem resultados mais próximos ao ótimo, as funções Booth e Easom são bidimensionais e as funções Alpine e Sphere são n-dimensionais de 10 e 6, respectivamente. As funções Griewank, Rastrigin e Rosenbrock que possuem resultados distantes do ideal são n-dimensionais todas com 50 dimensões. Dada as características dos algoritmos bio-inspirados, certas casos de teste levam mais iterações para encontrar o ótimo global. Logo, as soluções podem ser melhoradas com o aumento do número de iterações do algoritmo.

\footnotetext{
${ }^{3}$ Disponível em: https://drive.google.com/drive/folders/ 1sFq1T2sKbkmPp1DGrz1ISooR6uqy2Vng? usp=sharing
} 
No algoritmo ABC, as funções bidimensionais Booth (30,30\%) e Easom (41,32\%) possuem as menores taxas de ganho de desempenho, seguidas pelas funções Alpine $(35,59 \%)$ e Sphere $(36,65 \%)$. Os maiores ganhos de desempenho são para as funções Griewank (53,63\%), Rastrigin (63,34\%) e Rosenbrock (140,83\%). Todas possuem 50 dimensões. Observa-se que o ganho de desempenho aumenta conforme a dimensionalidade aumenta, proporcional a granularidade e as características do algoritmo.

$\mathrm{O}$ algoritmo PSO obteve taxas de ganho de desempenho significativas para as funções Alpine (301,01\%), Booth (602,84\%), Easom (289,20\%), Griewank (224,88\%), Rastrigin (276,75\%) e Rosenbrock (2007,388\%) e Sphere (1141,37\%).

É observável que as melhores médias variam o valor da população que a atingiu, tanto de soluções quanto de ganho de desempenho. Cada problema tem um algoritmo e uma população que é melhor aplicado ao problema.

\section{Considerações Finais}

Os algoritmos bio-inspirados correspondem a uma gama de estratégias eficientes para a solução de problemas de diversas áreas, desde uma simples análise de dados até problemas complexos como de geração de energia distribuída ou simulações de engenharia. $\mathrm{O}$ objetivo principal deste trabalho foi alcançado com a paralelização da biblioteca e o ganho de desempenho obtido nos testes dos algoritmos.

O ganho de desempenho obtido no PSO é superior ao ABC devido as características dos algoritmos. Enquanto o PSO é mais simples, com poucos saltos no código, o ABC possui mais estruturas condicionais que limitam a área paralela. A função Rosenbrock obteve a maior taxa de ganho de desempenho em ambos os algoritmos devido a granularidade. As características dessa função permitem que ela possa ser paralelizada.

As operações SIMD permitem explorar dos recursos da arquitetura vetorial. Dessa forma, é possível executar uma instrução sobre múltiplos dados utilizando os registradores vetoriais. Assim obtém-se um ganho de desempenho significativo como o apresentado neste trabalho.

Como trabalhos futuros buscamos, com o auxílio da comunidade de desenvolvedores, complementar a biblioteca paralela bio-inspirada com novos algoritmos paralelos.

\section{Referências}

Ignácio, A. A. V. and Ferreira, V. J. M. F. (2002). Mpi: uma ferramenta para implementação paralela. Pesquisa Operacional, 22:105 - 116.

Kennedy, J. and Eberhart, R. C. (2001). Swarm Intelligence. Morgan Kaufmann Publishers Inc., San Francisco, CA, USA.

Nievergelt, J., Gasser, R., Mäser, F., and Wirth, C. (1995). All the needles in a haystack: Can exhaustive search overcome combinatorial chaos? Springer Berlin Heidelberg, Berlin, Heidelberg.

Serapiao, A. (2009). Fundamentos de Otimização por Inteligência de enxames: Uma Visão Geral. Controle y Automacao, 20:271-304.

Yang, X.-S. (2010). Test problems in optimization. Engineering Optimization: An Introduction with Metaheuristic Applications. 